\title{
Surface of a catalyst in a gas phase
}

\author{
Yu Tang, Luan Nguyen, Yuting Li, Nan Wang, Franklin (Feng) Tao fl** $^{*}$ \\ ${ }^{\ddagger}$ Department of Chemical and Petroleum Engineering, University of Kansas, Lawrence, KS 66045 \\ "Department of Chemistry, University of Kansas, Lawrence, KS 66045
}

\begin{abstract}
Chemistry and structure of surface of a catalyst under a reaction condition is the crucial information for understanding catalytic mechanism since in many cases an authentic, active surface catalyzing a catalytic reaction is formed in a pretreatment or/and in a reaction between nominal catalyst and reactants. Ambient pressure X-ray photoelectron spectroscopy can be used to track surface of a catalyst under a reaction condition as the instrumentations in last decades have made characterization of catalyst surface in a gas phase at Torr pressure or higher possible. It can characterize surface chemistry of a catalyst including surface composition, surface phase and surface oxygen vacancies and other information under a reaction condition and track their evolutions when the reaction condition is changed to another.
\end{abstract}




\section{Introduction of significance of tracking surface of a catalyst}

Different from homogeneous catalysis, heterogeneous catalysis is a chemical process performed at the interface of a solid catalyst and reactants in a gas phase or a liquid phase, or even at the interface of a solid catalyst and liquid phase and gas phase of reactants. Compared to homogeneous catalysis in a liquid phase, a reaction in a gas phase, or a high temperature solid phase reaction, atoms of surface of a catalyst in heterogeneous catalysis must particulate into the chemical process of the catalytic reaction at a solid-liquid or solid-gas interface or a solid-liquidgas tri-phase region. Through involving the surface atoms of a catalyst, a different reaction pathway is taken in contrast to the reaction mechanism of intermolecular scattering potentially performed at a much higher temperature or/and higher pressure (if no a catalyst is used). The new pathway involving a catalyst typically exhibits a low overall activation barrier compared to the one without a catalyst. Compared to the chemical reaction processed at high temperature through molecular scattering (without a catalyst), significant amount of energy is saved for chemical transformations, which establishes the cornerstone role of catalysis in energy sciences. In addition, as a catalyst-involved pathway is kinetically favorable, heterogeneous catalysis has been the crucial chemical processes of chemical industries for efficient production of most materials used in industries and our daily lives ${ }^{1,2}$.

Unfortunately, this chemical process performed on surface of a catalyst is in fact quite complicated although only a shallow layer of surface atoms of a catalyst is involved in most cases. The complexity results from a few aspects. One is the variation of catalysts. There could be many catalysts active for the same chemical reaction. However, the catalytic mechanism of one catalyst at a molecular level in terms of a catalytic cycle of elementary steps could be very different from that of another. A thorough understanding of catalytic mechanism at a molecular 
level on a specific catalyst could never meant a understanding of the mechanism for the same chemical reaction catalyzed by another catalyst. Secondly, any catalytic cycle must involve the surface atoms of a catalyst. From surface science point of view, the surface processes of a catalytic cycle include surface adsorption of reactant molecules, dissociation of reactant molecules, a following coupling of different adsorbates on the same surface, formation of intermediate, and transformation of one intermediate to another intermediate of the next step through a transition state, formation of product molecules from the last intermediate, and desorption of product molecules, by which the catalyst surface is restored and be ready for next cycle of a catalytic event. Most of these elemental steps of a catalytic cycle involve atoms of the catalyst surface. Thus, the most complicated aspect of a catalytic reaction is the surface of the catalyst.

The complication in formation of an active catalyst was described as "catalyst digs its own bed"1. Definitely, The authentic active surface of a catalyst could be formed through pretreatment in a reactive gas such as $\mathrm{H}_{2}$ or $\mathrm{O}_{2}$ or/and a change of catalyst surface in the mixture of reactants of a catalytic reaction ${ }^{3}$. The crucial questions are how the surface of a catalyst changes under a pretreatment and under a reaction condition and what is the real surface structure catalyzing the chemical reaction. In search of catalysts through a trial-and-error manner, the surface structure of a catalyst before or after catalysis is typically considered for responding catalytic performances of a catalyst. However, the surface of a catalyst before or after pretreatment or during catalysis is likely different from the active surface which is catalyzing the chemical reaction. To rationally design a catalyst, a straightforward solution is to identify the authentic active surface of a catalyst to establish a correlation of this surface with its corresponding catalytic performance through in-situ/operando studies. As (a) the definitions of 
operando studies are quite different in literatures and (2) the difference of in-situ and operando studies was not well defined in literatures, here in-situ/operando study is used to describe a characterization of the surface of a catalyst while the catalyst is catalyzing a catalytic reaction. Typically, products of the catalytic reaction can be measured with an on-line mass spectrometer or even an on-line gas chromatography; a kinetics study can be performed simultaneously if necessary.

\section{Dynamic surface of catalyst in a gas or liquid phase of reactants}

In principle, in-situ/operando study should be done for all chemical processes if the information of reactants or/and products of a chemical reaction such as the surface of a catalyst is necessary for monitoring the production process, measuring yields during production process and understating the catalytic mechanism at a molecular level. Be specific, people could have performed in-situ/operando studies of heterogeneous catalysis by using electron-based techniques. However, most electron-based analytical techniques could not be applied to analyze structure or chemistry of a material under its existing condition (mostly ambient pressure at room temperature) and working condition. Thus, high vacuum environment is necessary for applying these electron-based techniques to analyze materials such as a catalyst. This by-pass strategy provides information of a material under ultra-clean environment, ultrahigh vacuum (UHV) which is distinctly different from its existing environment or working environment. If information achieved through characterization in UHV is the same as those of existing or working environment, the approach of UHV is fine. For example, the crystallographic structure of a mineral or a synthesized nanoparticle examined with TEM in UHV is the same as the authentic structure of the materials in air. However, for a chemical process performed on surface 
of a material such as a catalytic reaction performed at a solid-gas, solid-liquid, or even solidliquid-gas interface, the information achieved in UHV may not be the authentic information of the material in its existing or working environment ${ }^{4}$.

As mentioned above, typically the topmost atomic layer of a catalyst participates into the elementary steps of a catalytic cycle. The termination of crystallographic lattice at surface of a catalyst particle obviously makes atoms of the topmost layer highly active due to the low coordination numbers of the topmost layer. Surface energy of such a surface can be readily decreased by chemisorbing reactant molecules, which is called surface reconstruction ${ }^{2}$. One example is the surface oxidation of a metal, i.e. oxidation of most transition metal by air. More interestingly, surface structure of a metal could be changed by the existence of a gas phase of a reactant above the catalyst surface $e^{3,5,6}$. As shown in Figure 1, 0.1-1 Torr of CO gas above a model catalyst $\operatorname{Pt}(557)$ can break down the beautiful periodically packed stripe-like surface layer. On the other hand, the repulsion of $\mathrm{CO}$ molecules is increased along the increase of average number of $\mathrm{CO}$ molecules bound to a $\mathrm{Pt}$ atom. The increase of surface energy due to the formation of more under-coordinated Pt atoms can be over-compensated by decrease of energy of the surface through both chemisorbing more $\mathrm{CO}$ molecules at a higher pressure and releasing inter-molecular repulsion of adjacent $\mathrm{CO}$ molecules in a way called breaking the surface into nanoclusters. The overall consequence is the decrease of surface energy after breaking the stepped surface in gas of $\mathrm{CO}$ at a pressure or 0.1-1 Torr or higher. The breakdown of surface of $\operatorname{Pt}(557)$ is one example clearly revealing that the topmost surface layer can be readily changed by a reactive gas. 


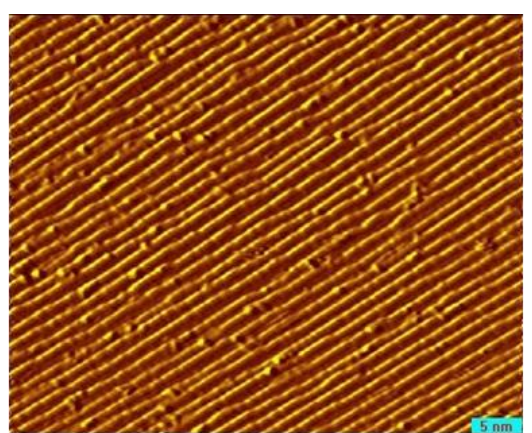

(a)

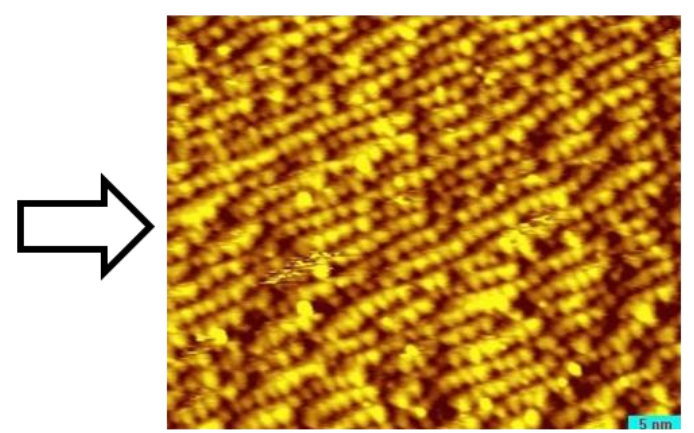

(b)

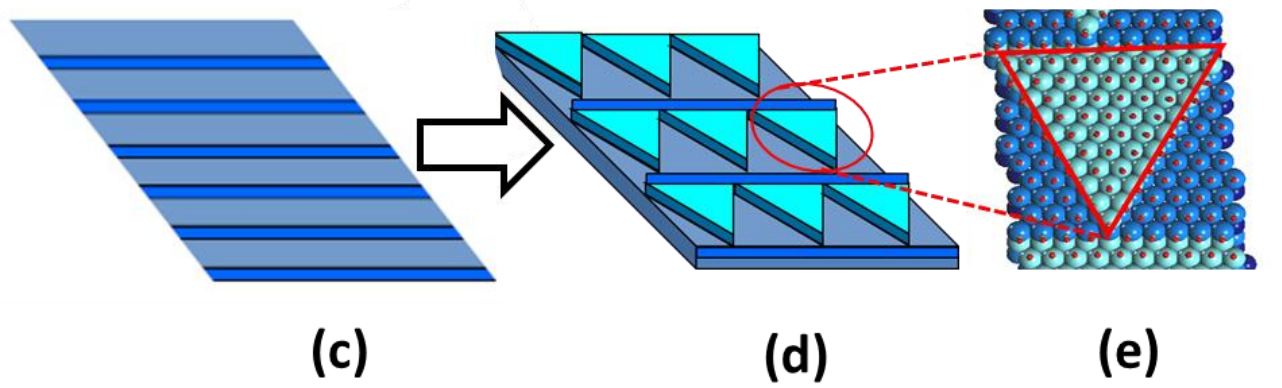

Figure 1. Surface restructuring driven by the pressure of reactants. (1) Surface of a stepped model catalyst in UHV $\left(2 \times 10^{-10}\right.$ Torr); on this surface $84 \% \mathrm{Pt}$ atoms (on terrace) and $16 \% \mathrm{Pt}$ atoms (on step edge) have coordination number of 9 and 7, respectively. (2) Breaking-up of the surface of stepped model catalyst to nanoclusters with a size of only $2.2 \mathrm{~nm}$; on this surface, more than $50 \% \mathrm{Pt}$ atoms have a coordination number of 7. (c) Schematic of $\mathrm{Pt}(557)$ surface in UHV. (d) Schematic of the formed Pt nanoclusters driven by CO at 0.1-1 Torr. (e) Surface of a nanocluster on which each $\mathrm{Pt}$ atom adsorbs one $\mathrm{CO}$ molecule.

Obviously the shallow layer of a catalyst surface could be restructured to some extent in one reactant or a mixture of reactants of a catalytic reaction. Such a reactant pressure-driven restructuring of a catalyst surface is well acceptable since the surface itself in fact can be considered as a chemical agent with high reactivity. Thus, the formation of an active surface could be an overall consequence of reaction conditions. For instance, chemical reaction of $\mathrm{Fe}_{2} \mathrm{O}_{3}$ with $\mathrm{CO}$ and $\mathrm{H}_{2}$ results in the formation of iron carbide active catalyst of Fischer-Tropsch synthesis ${ }^{7,8}$; pressure of reactants drives \ restructuring of surface such as formation of nanoclusters on hex- $\operatorname{Pt}(100)^{9}$ and $\operatorname{Pt}(557)^{5,6}$; reactants drive change of surface morphology of $\mathrm{Cu}$ nanoparticle supported on $\mathrm{ZnO}$ when gas phase is switched from $\mathrm{H}_{2}$ to a mixture of $\mathrm{H}_{2} \mathrm{O}$ and $\mathrm{H}_{2}$ 
to a mixture of $\mathrm{H}_{2}$ and $\mathrm{CO}^{10}$; controlled reduction of surface forms singly dispersed active bimetallic sites on a nonmetallic support ${ }^{11-14}$.

In terms of catalysis at solid-liquid interface, it is also true. The activity of carbonization of 1-arylethanols to 2-arylpropionic acids on a $\mathrm{Pd}$ nanoparticle in liquid is much higher than that on Pd-atom based molecular catalysts (homogeneous catalysis) ${ }^{15,16}$. It is hypothesized that the much higher catalytic activity of the Pd nanoparticles is due to the formation of palladium carbonyl at the solid-liquid-gas (CO) tri-phase region; the formation of the palladium carbonyl could be considered as a breaking-up of surface of a Pd nanoparticle in liquid of reactants, a type of dramatic restructuring of Pd nanoparticles in a liquid phase.

\section{Characterization of surface of a catalyst}

A fundamental understanding of catalytic mechanism requests to establish an intrinsic correlation between the authentic surface of a catalyst and its corresponding catalytic performance. The information of authentic surface can be basically divided into (1) adsorbate on surface of a catalyst and (2) atoms of surface of the catalyst. Adsorbates are the chemisorbed molecules of reactants, dissociated species of reactant molecules, spectator formed from strongly bonded reactant or its dissociated species, or intermediate formed at a low temperature but transformed to products at a high temperature. These coverages of these adsorbates except spectators strongly depend on temperature of the catalyst, pressure of the reactants A and B, and pressure ratio of reactants $\mathrm{A}$ and $\mathrm{B}$. In the case of catalysis at a solid-liquid or solid-liquid-gas interface, the dependences of coverage on temperature and pressure could be more complicated than those of catalyst in a gas phase since the solvent molecules of the liquid phase could change surface coverage largely and influence the diffusion of adsorbates on surface. In most cases, 
these adsorbates are organic moieties derived from reactant molecules; thus, infrared spectroscopy is a powerful technique to gather their vibrational signatures and then provide their identity.

Other than the adsorbates on a catalyst surface, information on surface of the catalyst is significant for understanding catalysis at a molecular level. Be specific, element identity, surface composition, oxidation state, packing of catalyst atoms of the surface layer of catalyst is fundamentally important information for having a picture of the authentic surface of a catalyst. In the case of adsorbates on surface, carbon-based adsorbates are distinctly different from the surface atoms of a catalyst in most cases; thus it is readily to distinguish adsorbates from surface of a catalyst. There is no issue of surface sensitively in the characterization of adsorbate in a catalyst surface. Unfortunately, however atoms of surface of a catalyst are the same or at least very similar to those of subsurface, deep layer, and bulk of a catalyst particle in terms of identify of elements. Thus, definitely surface sensitivity is one issue for characterization of catalyst surface. To clearly identify the chemistry and structure of surface of a catalyst, definitely a surface sensitive technique is necessary so that the change of the shallow layer can be reflected in the information volume of a technique to be chosen.

In terms of surface sensitivity, X-ray photoelectron spectroscopy is one of the main techniques. As shown from the well-known universal curve, photoelectrons with a kinetic energy of $20-1000 \mathrm{eV}$ have a mean free path of about $1 \mathrm{~nm}$ or so. $\mathrm{Al} \mathrm{K} \alpha(\mathrm{hv}=1486.3 \mathrm{eV})$ or $\mathrm{Mg} \mathrm{K} \alpha$ ( $\mathrm{h} v=1253.4 \mathrm{eV}$ ) cover sub-shells of most elements which exhibit the strongest photoemission cross section such as 1 s subshell of carbon atoms, $2 \mathrm{p}$ of silicon, $3 \mathrm{~d}$ of palladium and $4 \mathrm{f}$ of platinum. In addition, available single crystal, quartz can monochromate the radiation lines of Al target and thus provide a pure $\mathrm{Al} \mathrm{K} \alpha$ radiation with a width of only $0.3 \mathrm{eV}$. Thus, $\mathrm{Al} \mathrm{K} \alpha$ has been 
the workhorse of surface analysis of various materials in research labs. The high resolution energy analyzer designed by Kai Siegbahn has made Al Ka capable of distinguishing chemical states of the same atoms in a slight different chemical environment since $1960 \mathrm{~s}^{17-20}$. Thus, XPS was also named as electron spectroscopy for chemical analysis by Kai Siegbahn in 1960s ${ }^{17-22}$.

\section{Track surface of a material in a gas or liquid phase}

Typically, surface chemistry of a material can be readily characterized with XPS in a high vacuum or ultrahigh vacuum environment. For many chemical process including heterogeneous catalysis, characterization of surface chemistry of a material under its existing or working environment is necessary. Kai Siegbahn and his group have performed pioneer work in characterization of surface of materials in gas and even liquid phase in $1970 \mathrm{~s}^{22-24}$. The collection of photoelectrons generated through photoexcitation of synchrotron was enhanced by focusing lenses installed before an energy analyzer by Ogletree et $\mathrm{al}^{25,26}$; gas leaking through aperture was being pumped through differentially pumping stages of energy analyzer (also called ambient pressure energy analyzer). These instrumentations made collection of enough photoelectrons possible when a sample is in a gas phase at sub-torr to torr pressure. By using lab-based X-ray source, particularly an X-ray gun with the enhanced photo flux density using a micro-focus electron beam, good number of photoelectrons per $\mathrm{mm}^{2}$ per second can be generated for surface analysis of a material in a gas phase ${ }^{27}$.

\section{Application of ambient pressure X-ray photoelectron spectroscopy technique to track surface of catalyst under reaction conditions}


In fundamental understanding of catalytic mechanism at a molecular level, kinetics studies of a catalytic reaction is typically necessary. A fixed-bed flow reactor can be used to perform a catalytic reaction in a kinetics control regime. Typically, the conversion of reactant is lower than $10 \%-20 \%$. The Arrhenius plot of $\ln$ [reaction rate] versus $1 / T(\mathrm{~K})$ allows to calculate the overall activation barrier through the slope of a straight line fitted into the data points of this Arrhenius plot. Ideally, surface chemistry of a catalyst in the temperature range of kinetics studies during catalysis should be collected and then be correlated with the measured kinetics for fundamental understanding of the catalytic mechanism since the surface simultaneously identified during kinetics studies is the authentic surface resulting in the measured kinetics of the catalytic pathway.

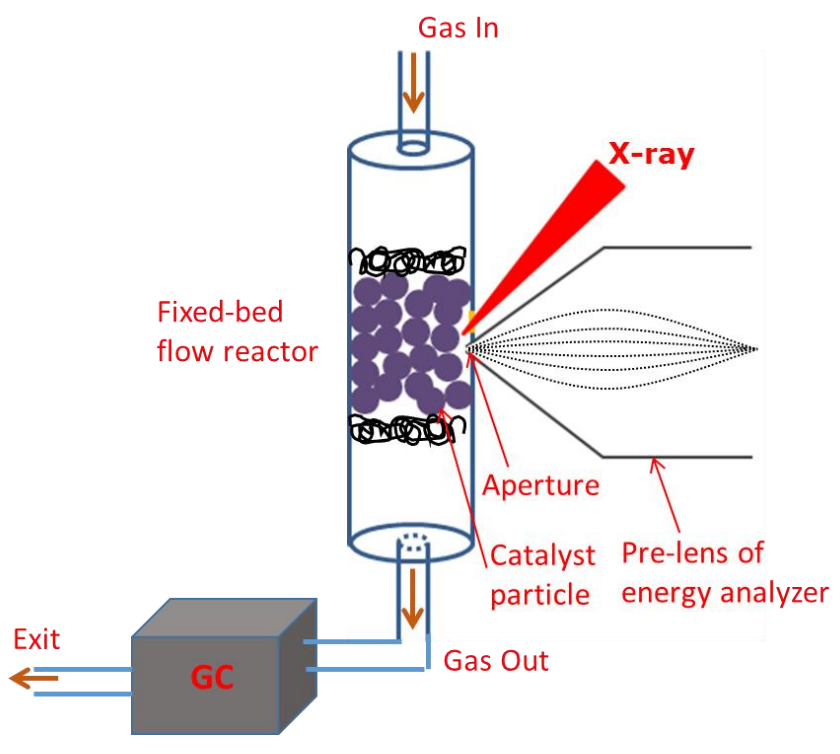

Figure 2. Schematic of ideal in-situ/operando studies of a catalyst surface during catalysis (under a kinetics controlled regime) performed in a fixed-bed flow reactor. With this ideal setup, the kinetic studies of a catalytic reaction on a catalyst can be directly correlated with the corresponding catalytic performance during catalysis. 
Figure 2 schematically shows the ideal setup of the in-situ/operando studies using ambient pressure XPS. In reality, however it is challenging to collect data from the catalyst nanoparticles packed in the catalyst bed in the middle of a fixed-bed flow reactor. One reason is the loosely packed catalyst particles could be easily suck to the aperture and thus block the travel of photoelectrons, probably resulting in the impossibility of collection of photoelectrons for analysis. In addition, small catalyst particles could pass through the aperture and then fall down to the pre-lens and then scatter with rotator of turbo molecular pumps. Another reason is the limit of pressure of gas in which photoelectrons could travel through. The upper limit of the pressure is decided by a few factors to be discussed in the following Section 5.2. A compromised method to track surface of a catalyst under a reaction condition by using ambient pressure X-ray photoelectron technique is to load catalysts on surface of an inert substrate and locate the sample in a flowing gas environment although I expect that our on-going effort of my group will be able to perform simultaneous analysis of surface of catalyst particles while catalysis on the surface of catalyst particles in a fixed-bed flow reactor is being performed. The following sections will describe how a compromised method was realized for achieving information of surface of a catalyst under reaction conditions.

\subsection{Create a gas environment of reactants in which a catalyst is located}

AP-XPS has been widely used to the analyses of surface of electrodes or materials in a static gaseous environment such as surface of electrodes of fuel cell and battery and even surface of photocatalyst under working conditions. Compared to the applications to electrochemical 
process, for in-situ/operando studies of a catalyst during catalysis a local gaseous environment with certain flow rate is necessary. A gas environment can be readily created by filling gas to a UHV chamber which typically has a size of 5-20 liters. However, such a gaseous environment is static although the gas is being pumped through the aperture interfaced at the chamber and prelens of the energy analyzer. Comparted to this nearly static gas environment (Figure 3a), a gas environment with a much higher flow rate can be created through installation of a reaction cell (Figure 3b). Typically, the volume of a reaction cell is $20-30 \mathrm{ml}^{28}$. With the same pumping capacity provided by the same aperture and same two turbo pumps installed at the pre-lens stage, a pumping rate is typically $2-5 \mathrm{ml} / \mathrm{min}$ which is much larger than the pumping rate $(0.01-0.1$ $\mathrm{ml} / \mathrm{min}$ ) of gas filled to a UHV chamber with a volume of 5-20 liters.

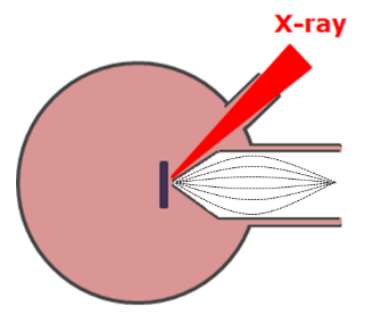

(a)

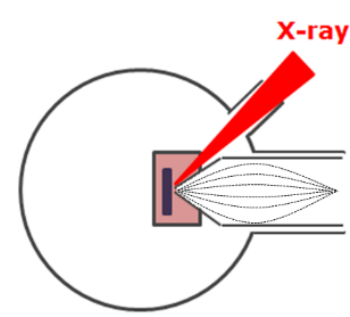

(b)

Figure 3. Schematics of in-situ/operando studies of catalyst in a gas phase under a reaction condition. (a) A method of filling gas to a UHV chamber with a volume of 5-20 liters. (b) A method of filling reactant gases to a small reaction cell with a volume of $20-30 \mathrm{ml}$.

\subsection{Precise control of sample-aperture distance}

For photoelectrons generated from surface region of a catalyst in a gas phase, they experience two types of attenuations. One is the attenuation in solid by inelastic scattering when they travel through atomic layers between the source atomic layer of photoelectrons and the topmost layer of the catalyst surface. This attenuation is the same as that of photoelectrons traveling through surface region of a solid when the sample is in UHV. Another is the 
attenuation in a gas phase they travel from the surface to an aperture which interfaces the gaseous environment around the catalyst and vacuum environment of the pre-lens stage. The second attenuation is determined by both (1) the pressure of the gas between surface of a catalyst and the pumping aperture, (2) the sample surface-aperture distance, and (3) the type of gas around the sample. Figure 4a schematically presents a sample located in gas phase and an aperture at the interface of gas phase and vacuum of the pre-lens stage. The following equation clearly shows that the attenuation is exponentially proportional to thickness of the gas in terms of the distance between surface of a catalyst and the aperture and to pressure of the gas. $\frac{I_{p}}{I_{o}}\left(E_{K}, p\right)=e^{\frac{-z \sigma_{E_{K}} p}{\kappa T}}, 29$ called attenuation or the fraction of the collected photoelectrons $\left(\frac{I_{p}}{I_{o}}\right)$ of a particular kinetic energy $\left(E_{K}\right)$, exponentially decreases along the increases of sample-aperture distance in terms of the travel distance of photoelectrons in a gas phase and the pressure of gas $(p)$ they travel through. In this equation, $k$ is the Boltzmann constant; $T$ is the temperature of gas, and $\sigma$ is the electron scattering cross section for a particular gas. A higher pressure leads to a much larger attenuation since a photoelectron has to inelatically scatter with more molecules. This is also true for the thickness of the gas phase. Figure $4 \mathrm{~b}$ plots the exponentially decay of collection rate as a function of the sample-aperture distance. Obviously, at a higher pressure the decay is faster. For instances, obviously the decay of photoelectrons in 1 or 10 Torr of $\mathrm{H}_{2} \mathrm{O}$ is $16 \%$ and $85 \%$ at a distance of $0.5 \mathrm{~mm}$. Figure $4 \mathrm{~b}$ justifies why in most cases the chosen pressure of in-situ/operando studies in literature are one torr or sub-torr 


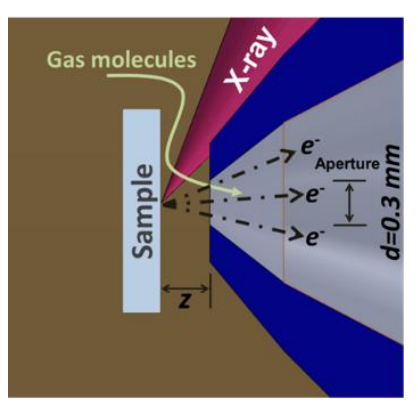

(a)

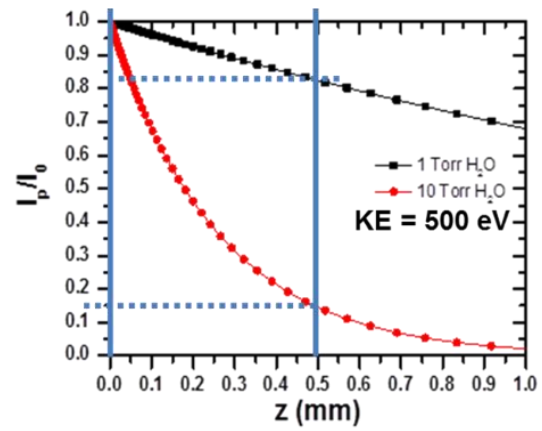

(b)

Figure 4. Dependence of collection rate of photoelectrons on the sample-aperture distance, and collection area, pressure of reactant gas. (a) Schematic showing the configuration of sample with a distance of $z$ from aperture with an internal diameter of $d$. (b) Plot of collection rate (Io/I) as a function of sample-aperture distance in water vapor at 1 Torr and 10 Torr.

Empirically, the distance between sample surface and aperture is approximately the mean free path of photoelectrons in the gas at a pressure $p$. Certainly, either a higher pressure of gas or a lower kinetic energy of photoelectrons gives a shorter mean free path of photoelectrons in a gas phase. For instance, the mean free path of an electron with kinetic energy of $500 \mathrm{eV}$ in 1 Torr of nitrogen is about 1-2 mm. For collecting photoelectrons, a sample-aperture distance typically is requested to remain at about $1 \mathrm{~mm}$. It is noted that to remain the pressure of gas above sample surface at $90 \%$ of the measured pressure of gas phase on the wall of a cell or of a UHV chamber, it is necessary to remain the diameter of the aperture at or smaller than $(\mathrm{d}<2 \mathrm{z})$. Due to this requirement of the fluid dynamics, increase of collection rate of photoelectrons $\left(\mathrm{I} / \mathrm{I}_{\mathrm{o}}\right)$ through decrease of sample-aperture distance (z) will be balanced by decrease of collection ratio through a decrease of collection area $\left(1 / 4 \pi \mathrm{d}^{2}\right)$. An optimum distance for largest collection rate is typically achieved by tuning the sample-aperture distance when an aperture of $0.8-1 \mathrm{~mm}$ is used for 1 Torr.

\subsection{Flux density of $\mathrm{X}$-ray radiation and collection rate of photoelectrons}


An important feature of AP-XPS is the small collection area which is typically $0.8-1 \mathrm{~mm}$ for sub-Torr to 1 Torr pressure. For 10 Torr gas or higher, a small aperture with a diameter of 0.2 or $0.3 \mathrm{~mm}$ is typically used. To collect reasonable number of photoelectrons through an aperture of $0.3 \mathrm{~mm}$, an X-ray with a high flux density in a unit of the number of photons per $\mathrm{mm}^{2}$ per second received on sample is the key parameter. By using the same aperture, an X-ray beam with high flux density obviously can excite more photoelectrons per second. It is noted that flux in a unit of number of photons per second of an X-ray beam is not the parameter to judge the number of photoelectrons to be collected from aperture although the flux could have been used to evaluate the number of photoelectrons to be collected at the entrance of energy analyzer in some cases of vacuum XPS. In addition, flux density is not strongly related to the upper limit of the pressure at which a spectrum can be collected. But X-ray beam with low flux density will request more time to collect a spectrum than X-ray beam with high flux density. As long as there is no beam damage or beam-induced chemistry, a high flux density requests less scanning time. In other words, the scanning time of spectrum with the same signal-to-noise ratio strongly depends on the flux density. Collection of a spectrum in a timescale of a few second is not necessary if the kinetics of evolution of surface chemistry at a time scale of seconds is not the topics to study.

\subsection{Track surface of $\mathrm{Ag}$ thin film in $\mathrm{N}_{2}$}

A lab-based AP-XPS was installed at University of Notre Dame through the collaboration of Tao and Specs Surface Nano GmbH (Figures 5a and 5b). Now it is used by a user group at Notre Dame. $\mu$-focus 600 X-ray source was used on the system. Inset of Figure $5 \mathrm{c}$ is the photo of aperture and sample a distance of about $0.3 \mathrm{~mm}$. Ag 3d spectrum with reasonable signal/noise ratio was collected from Ag thin film in 25 Torr $\mathrm{N}_{2}$ in about 1-2 minutes $^{27}$ (Figure 5d). 


\section{Examples of information of a catalyst surface under reaction condition AP-}

\section{XPS can provide}

The following sub-sections will exemplify the information of catalyst surfaces under reaction condition that AP-XPS could provide. It is noted that AP-XPS, particularly synchrotron-based AP-XPS has largely used in characterizing surface of electrodes of fuel cells and battery and other materials. Due to the limited space and the focus topic, here only some examples of APXPS of using AP-XPS to heterogeneous catalysis in gas phase of reactants under reaction conditions were chosen as examples. To catch the surface characterization of electrons and other materials with AP-XPS, please refer to research articles or reviews published in literatures.
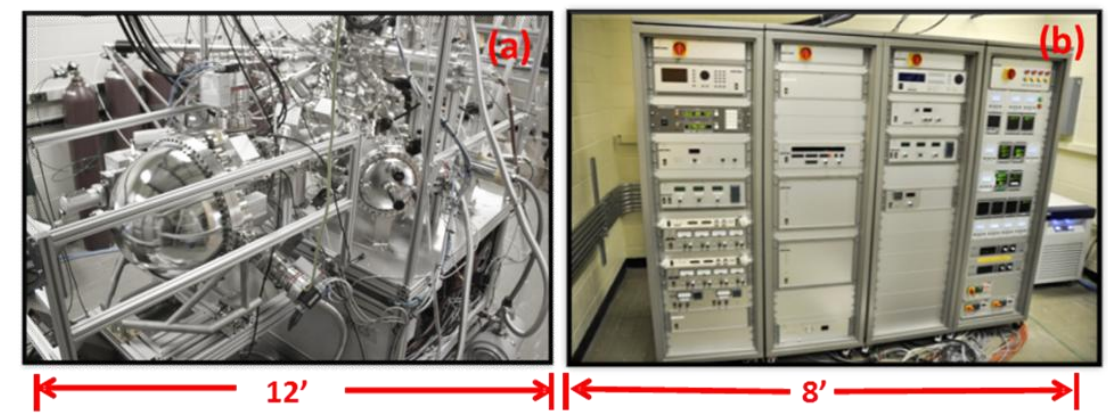

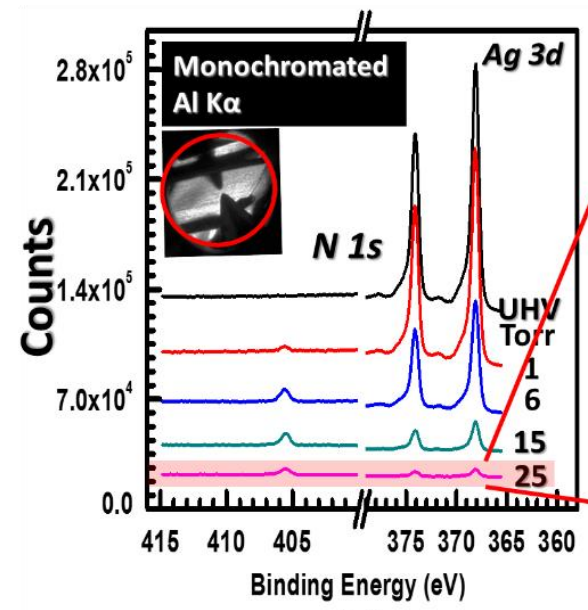

(c)

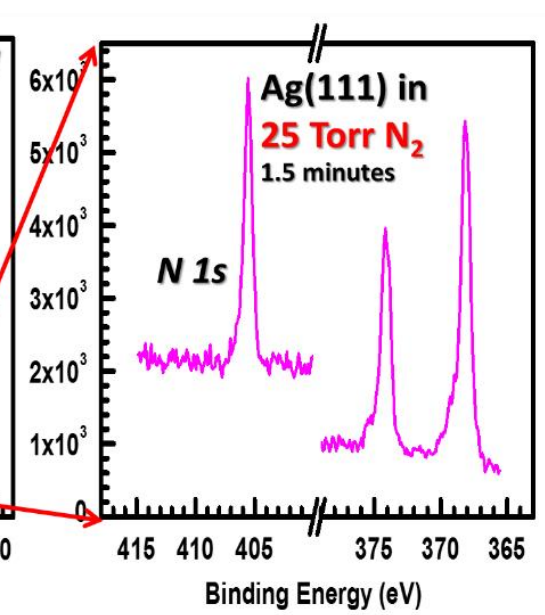

(d)

Figure 5. A lab-based ambient pressure X-ray photoelectron spectrometer and its function. (a) Monochromated X-ray source, ambient pressure energy analyzer, and XPS chamber and sample preparation chamber. (b) Electronics of the AP-XPS system. (c) and (d) Photoemission feature of $\mathrm{Ag} 3 \mathrm{~d}$ of silver film in UHV and gas of $\mathrm{N}_{2}$ in a reaction cell at different pressures. 


\subsection{Track transformation of surface phase under reaction condition}

An authentic active phases of catalyst surface could be formed through a pretreatment of a catalyst precursor in a gas typically $\mathrm{H}_{2}$ or $\mathrm{O}_{2}$ and a reaction between a "nominal catalyst" and one or all reactants of a catalytic reaction. For instance, $\mathrm{H}_{2}$ is typically used to reduce a prepared "catalyst precursor" to form a metal catalyst. This simple reduction can be predicted readily. Oxidation of a catalyst precursor to an oxide can be expected as well. Other than these predictable phase formation of metal in $\mathrm{H}_{2}$ and oxide in $\mathrm{O}_{2}$, it is hard to predict a potential transformation of a surface phase of a catalyst in the mixture of reactants of a catalytic reaction. In these cases, track of surface of such a catalyst in the mixture of reactants is necessary.

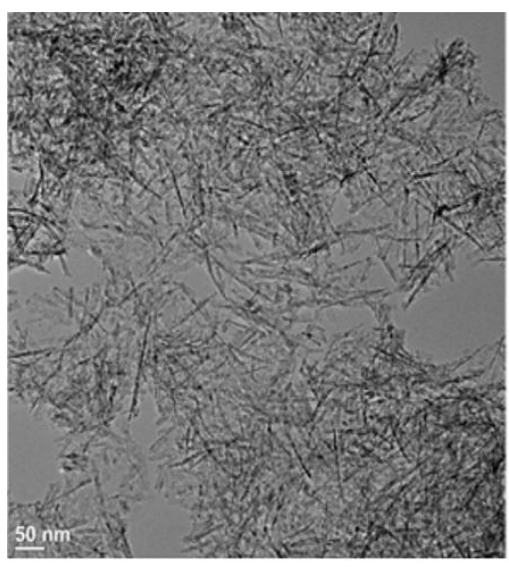

(a)

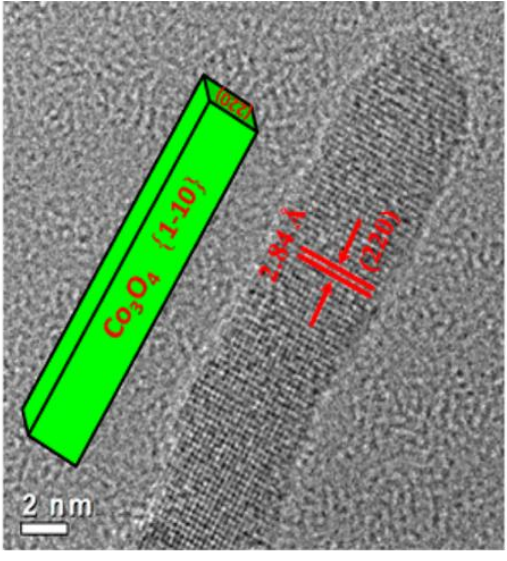

(b)

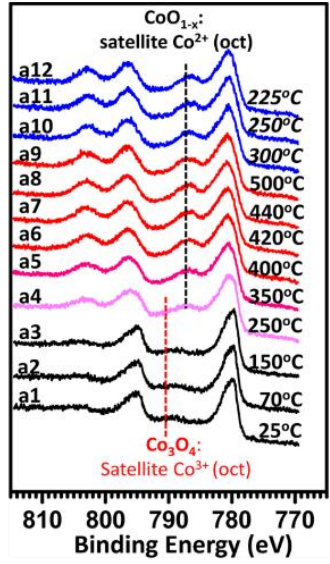

(c)

Figure 6. Characterization of surface of $\mathrm{Co}_{3} \mathrm{O}_{4}$ nanorods in the mixture of $\mathrm{CO}$ and $\mathrm{NO}$ at Torr pressure in the temperature range of $25^{\circ} \mathrm{C}-500^{\circ} \mathrm{C}$ (annealing) and $500^{\circ} \mathrm{C}-225^{\circ} \mathrm{C}$ (cooling). (a) Large scale TEM image of $\mathrm{Co}_{3} \mathrm{O}_{4}$ nanorods. (b) High resolution TEM image of a $\mathrm{Co}_{3} \mathrm{O}_{4}$ nanorod. (c) Evolution of photoelectron feature of $\mathrm{Co}_{2} \mathrm{pCo}_{3} \mathrm{O}_{4}$ nanorods in the mixture of $\mathrm{CO}$ and $\mathrm{NO}$ at different temperature in $25^{\circ} \mathrm{C}-500^{\circ} \mathrm{C}$ (annealing) and $500^{\circ} \mathrm{C}-225^{\circ} \mathrm{C}$ (cooling). 
$\mathrm{Co}_{3} \mathrm{O}_{4}$ nanorods with preferentially exposed (110) were synthesized (Figures $6 \mathrm{a}$ and $\left.6 \mathrm{~b}\right)^{30-32}$. In $\mathrm{Co}_{3} \mathrm{O}_{4}, \mathrm{Co}^{3+}$ and $\mathrm{Co}^{2+}$ coordinate with 6 and 4 oxygen atoms through octahedral and tetrahedral coordinations, respectively. The coordination of $\mathrm{Co}^{3+}$ with six oxygen atoms gives a very weak satellite peak of Co $2 \mathrm{p}_{3 / 2}$ at $789.6 \mathrm{eV}^{31,32}$. Different from photoemission feature of Co $2 \mathrm{p}_{3 / 2}$ of $\mathrm{Co}_{3} \mathrm{O}_{4}$, a strong satellite peaks of $\mathrm{Co}^{2+}$ in a tetrahedral coordination with four oxygen atoms of $\mathrm{CoO}$ is at $786.8 \mathrm{eV}^{31,32}$. Only $\mathrm{Co}^{2+}$ of $\mathrm{CoO}\left(\right.$ nor $\mathrm{Co}^{2+}$ of $\left.\mathrm{Co}_{3} \mathrm{O}_{4}\right)$ is in a tetrahedral coordination. By checking the photoemission feature of Co $2 \mathrm{p}_{3 / 2}$ at $25^{\circ} \mathrm{C}$ (Figure $6 \mathrm{c}$ ), the assynthesized $\mathrm{Co}_{3} \mathrm{O}_{4}$ is a spinel phase as what we predicted. The surface phase of $\mathrm{Co}_{3} \mathrm{O}_{4}$ is remained in the mixture of $\mathrm{CO}$ and $\mathrm{NO}$ at Torr pressure up to $150^{\circ} \mathrm{C}$. However, a satellite peak of Co $2 \mathrm{p}_{3 / 2}$ at $786.8 \mathrm{eV}$ is clearly observed at $250^{\circ} \mathrm{C}$ when the nominal catalyst $\mathrm{Co}_{3} \mathrm{O}_{4}$ was annealed to $250^{\circ} \mathrm{C}$. It suggests that the surface phase, $\mathrm{Co}_{3} \mathrm{O}_{4}$ was partially transformed to $\mathrm{CoO}_{1-\mathrm{x}}$ at $250^{\circ} \mathrm{C}$. Along the increase of the reaction temperature, the ratio of intensity of the satellite peak of Co $2 \mathrm{p}_{3 / 2}$ to its main peak is increased and reaches maximum at $500^{\circ} \mathrm{C}$. This in-situ track of catalyst surface in the temperature range of $250^{\circ} \mathrm{C}-500^{\circ} \mathrm{C}$ suggests that the active phase of the nominal catalyst is cobalt monoxide under the reaction condition.

\section{Track evolution of surface composition of bimetallic catalysts}

Bimetallic catalyst is one main categories of catalysts. By adding another metal, the catalytic performance of a host metal can be tuned largely through several effects including electronic effect, ligand (or called geometry) effect, and lattice strain effect. It is noted that the surface composition of a bimetallic catalyst is one of the key descriptors for understanding catalytic performance of a bimetallic catalyst. As the two constituting metals typically have different surface energy and could exhibit quite different adsorption energy for a reactant molecule, 
surface composition of an as-prepared bimetallic catalyst could be different from that in UHV or different from one condition to another. From thermodynamic point of view, the surface composition is decided by a compromised balance of adsorption energy of molecules on metal atoms and surface energy of metal atoms ${ }^{33,34}$.

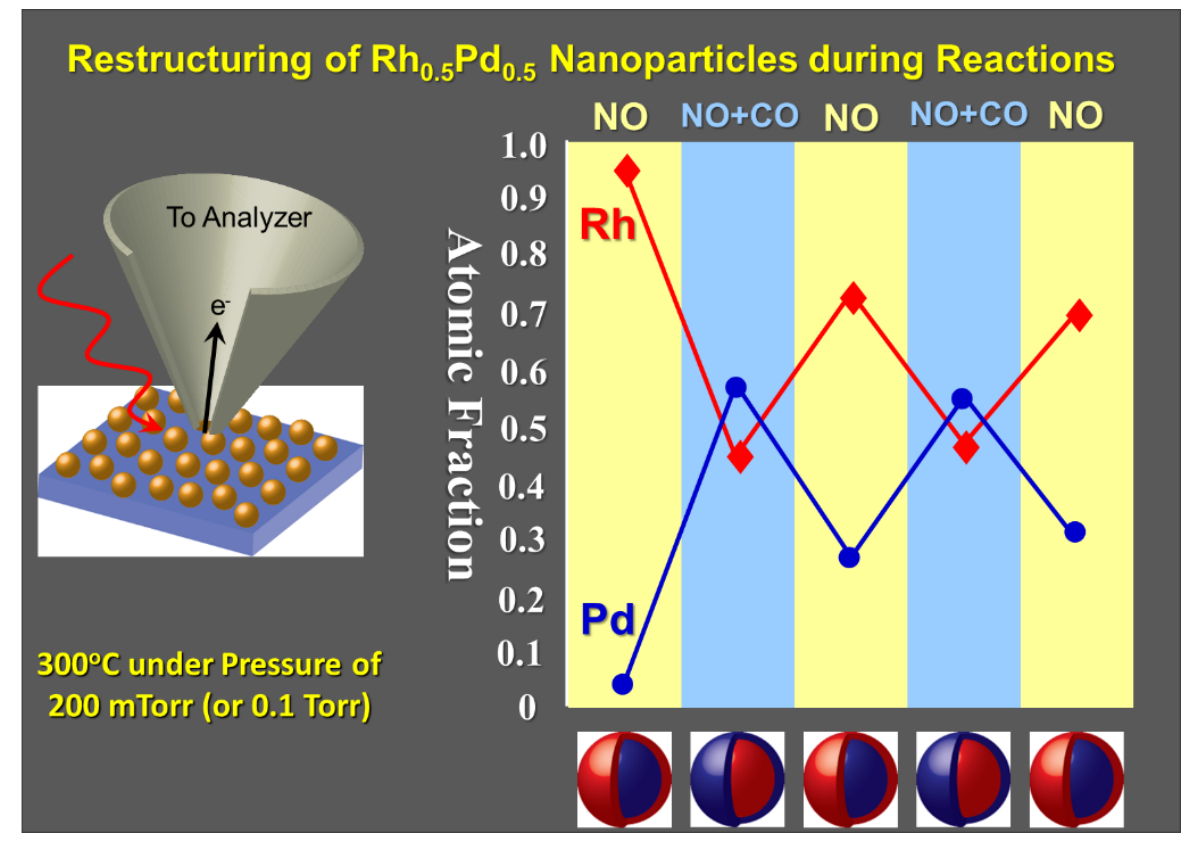

Figure 7. Measurements of atomic fractions of $\mathrm{Rh}$ and $\mathrm{Pd}$ in the surface layers of $\mathrm{Rh}_{0.5} \mathrm{Pd}_{0.5}$ bimetallic nanoparticles supported on silicon wafer in a gas phase at Torr pressure and evolution of compositions of the surface layers under different reaction conditions.

AP-XPS can be used to measure surface composition under different reaction conditions. As shown in Figure 7 based on AP-XPS studies of $\mathrm{Rh}_{0.5} \mathrm{Pd}_{0.5}$ bimetallic nanoparticles in different gases, surface composition of $\mathrm{Rh}_{0.5} \mathrm{Pd}_{0.5}$ bimetallic nanoparticles change if the gas environment is switched from a reducing gas to oxidizing ${ }^{33,34}$. The surface composition can be tuned readily by using different reactant gases. This evolution of surface compositions can be readily tracked 
with AP-XPS. A similar compositional restructuring of a catalyst surface was observed on other catalyst system including Rh-Pt ${ }^{33,34}$.

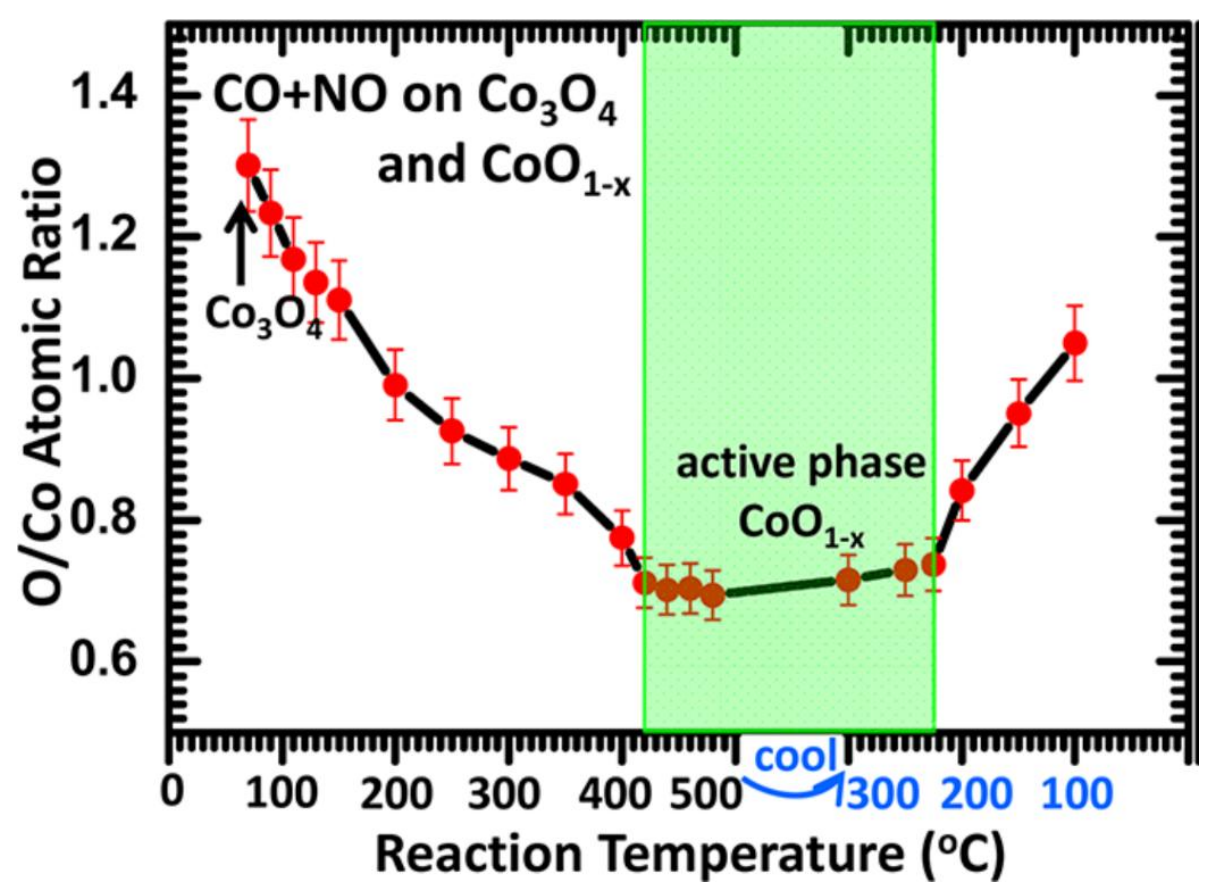

Figure 8. Measurements of atomic fractions of $\mathrm{O} / \mathrm{Co}$ of the surface layers of nominal catalyst, $\mathrm{Co}_{3} \mathrm{O}_{4}$ nanorods in the mixture of $\mathrm{CO}$ and $\mathrm{NO}$ at Torr pressure and evolution of the $\mathrm{O} / \mathrm{Co}$ ratio as a function of reaction temperature $\left(80^{\circ} \mathrm{C}-500^{\circ} \mathrm{C}\right.$ and $\left.500^{\circ} \mathrm{C}-100^{\circ} \mathrm{C}\right)$.

\section{Track surface oxygen vacancies of catalyst under reaction conditions}

Oxygen vacancies can be formed on many transition metal oxides in a gas phase, particularly these reducible oxides such as $\mathrm{CeO}_{2}, \mathrm{Co}_{3} \mathrm{O}_{4}, \mathrm{CoO}, \mathrm{TiO}_{2}, \mathrm{MnO}_{2}$ and $\mathrm{Fe}_{3} \mathrm{O}_{4}{ }^{35-41}$. Density of oxygen vacancies and how it enhances at different conditions are an important piece of information for understanding catalytic mechanism. Reducible oxide could be a catalyst or a part of a catalyst. The O/M atomic ratio (M: metal element) can be readily measured with XPS. Notably, in the gas phase of a reducing or oxidizing reactant or a mixture of all reactants of a 
catalytic reaction, the surface composition in terms of the density of oxygen vacancies could be quite different from that of an as-synthesized catalyst. AP-XPS is the right technique to measure the density of oxygen vacancies under reaction conditions and track its change under different reaction conditions. As shown in Figure 8, the O/Co ratio was readily measured at difference temperatures ${ }^{30}$. Progressive loss of oxygen atoms along the increase of reaction temperature in the mixture of $\mathrm{CO}$ and $\mathrm{NO}$ was clearly revealed. The nature of nonstoichiometric $\mathrm{CoO}_{1-\mathrm{x}}$ was discovered by AP-XPS studies under reaction condition at $420^{\circ} \mathrm{C}-500^{\circ} \mathrm{C}$ and $500^{\circ} \mathrm{C}-225^{\circ} \mathrm{C}$ in the mixture of $\mathrm{CO}$ and $\mathrm{NO}$.

\section{Summary}

The participation of atoms of catalyst surface into the chemical process of heterogeneous catalysis requests the track of surface chemistry and structure of catalyst under a reaction condition since the gas phase of a reactant or a mixture of all reactants of a catalytic reaction could largely change surface of the catalyst including surface composition, oxidation state, surface phase in contrast to those of as-synthesized catalysts. The surface of a catalyst in a gas phase under a reaction condition and its evolution from one condition to another can be measured or tracked with AP-XPS since the development of AP-XPS technique in the last three decades pioneered by Kai Siegbahn and his group and the recent advances has offered the capability of characterizing surfaces of catalysts under reaction conditions in Torr pressure range. This technique has been used to explore surfaces of catalysts under reaction conditions. These explorations allows for establishing a correlation between surface chemistry of a catalyst and its corresponding catalytic performance. 


\section{Acknowledgments}

FT acknowledges the financial support from Chemical Sciences, Geosciences and Biosciences

Division, Office of Basic Energy Sciences, Office of Science, U.S. Department of Energy under

Grant No. DE-SC0014561, the National Science Foundation Career Award NSF-CHE-

14162121, National Science Foundation under the grant No. NSF-OIA-1539105 and National

Science Foundation under the grant No. CBET-1264798.

\section{References}

(1) Ertl, G.; Knözinger, H.: Handbook of Heterogeneous Catalysis; Wiley-VCH, 2008.

(2) Somorjai, G. A.; Li, Y.: Introduction of Surface Chemistry and Catalysis; Wiley-VCH, 2010.

(3) Tao, F.; Salmeron, M. In Situ Studies of Chemistry and Structure of Materials in Reactive Environments. Science 2011, 331, 171-174.

(4) Tao, F. F.; Crozier, P. Atomic-Scale Observations of Catalyst Structures under Reaction Conditions and during Catalysis. Chemical Reviews 2016, DOI: 10.1021/cr5002657.

- This review focuses on structures of catalyst nanoparticles including surface and bulk, their characterizations, and how a potential restructuring could shape their catalytic performances. This article emphasizes how challenging in tracking surface structure of a catalyst at atomic or nano scale and how significant this challenging task is for understanding catalysis at a molecular scale.

(5) Tao, F.; Dag, S.; Wang, L.-W.; Liu, Z.; Butcher, D. R.; Bluhm, H.; Salmeron, M.; Somorjai, G. A. Break-Up of Stepped Platinum Catalyst Surfaces by High CO Coverage. Science 2010, 327, 850-853.

- This article revealed the restructuring of surfaces of single-crystal model catalysts driven by gas phase at Torr pressure. This work suggested that potential restructurings on other metals are possible. Whether there is a restructuring is determined by the competition among metal-metal interaction on surface, metal-molecule binding, and intermolecular repulsion of the adsorbed molecules.

(6) Zhu, Z.; Tao, F.; Zheng, F.; Chang, R.; Li, Y.; Heinke, L.; Liu, Z.; Salmeron, M.; Somorjai, G. A. Formation of Nanometer-Sized Surface Platinum Oxide Clusters on a Stepped Pt(557) Single Crystal Surface Induced by Oxygen: A High-Pressure STM and Ambient-Pressure XPS Study. Nano Letters 2012, 12, 1491-1497.

(7) de Smit, E.; Weckhuysen, B. M. The renaissance of iron-based Fischer-Tropsch synthesis: on the multifaceted catalyst deactivation behaviour. Chemical Society Reviews 2008, 37, 2758-2781. 
(8) Sun, Z.; Sun, B.; Qiao, M.; Wei, J.; Yue, Q.; Wang, C.; Deng, Y.; Kaliaguine, S.; Zhao, D. A General Chelate-Assisted Co-Assembly to Metallic Nanoparticles-Incorporated Ordered Mesoporous Carbon Catalysts for Fischer-Tropsch Synthesis. Journal of the American Chemical Society 2012, 134, 17653-17660.

(9) Tao, F.; Dag, S.; Wang, L.-W.; Liu, Z.; Butcher, D. R.; Salmeron, M.; Somorjai, G. A. Restructuring of hex-Pt(100) under CO Gas Environments: Formation of 2-D Nanoclusters. Nano Letters 2009, 9, 2167-2171.

(10) Hansen, P. L.; Wagner, J. B.; Helveg, S.; Rostrup-Nielsen, J. R.; Clausen, B. S.; Topsoe, H. Atom-resolved imaging of dynamic shape changes in supported copper nanocrystals. Science 2002, 295, 2053-2055.

(11) Zhang, S.; Nguyen, L.; Liang, J.-X.; Shan, J.; Liu, J.; Frenkel, A. I.; Patlolla, A.; Huang, W.; Li, J.; Tao, F. Catalysis on singly dispersed bimetallic sites. Nature Communications 2015, 6.

(12) Tao, F. F.; Shan, J.-j.; Nguyen, L.; Wang, Z.; Zhang, S.; Zhang, L.; Wu, Z.; Huang, W.; Zeng, S.; Hu, P. Understanding complete oxidation of methane on spinel oxides at a molecular level. Nature Communications 2015, 6.

(13) Wang, L.; Zhang, S.; Zhu, Y.; Patlolla, A.; Shan, J.; Yoshida, H.; Takeda, S.; Frenkel, A. I.; Tao, F. Catalysis and In Situ Studies of Rh-1/Co3O4 Nanorods in Reduction of NO with H-2. Acs Catalysis 2013, 3, 1011-1019.

(14) Zhang, S.; Shan, J.-j.; Zhu, Y.; Frenkel, A. I.; Patlolla, A.; Huang, W.; Yoon, S. J.; Wang, L.; Yoshida, H.; Takeda, S.; Tao, F. WGS Catalysis and In Situ Studies of CoO1-x, PtCon/Co3O4, and PtmCom '/CoO1-x Nanorod Catalysts. Journal of the American Chemical Society 2013, 135, 8283-8293.

(15) Jayasree, S.; Seayad, A.; Chaudhari, R. V. Highly active supported palladium catalyst for the regioselective synthesis of 2-arylpropionic acids by carbonylation. Chemical Communications 1999, 1067-1068.

(16) Davies, I. W.; Matty, L.; Hughes, D. L.; Reider, P. J. Are heterogeneous catalysts precursors to homogeneous catalysts? Journal of the American Chemical Society 2001, 123, 1013910140.

(17) Fahlman, A.; Siegbahn, K. ESCA METHOD USING MONOCHROMATIC X-RAYS AND A PERMANENT MAGNET SPECTROGRAPH. Arkiv for Fysik 1966, 32, 111-\&.

(18) Hamrin, K.; Johansson, G.; Fahlman, A.; Nordling, C.; Siegbahn, K.; Lindberg, B. Structure studies of sulphur compounds by ESCA. Chemical Physics Letters 1968, 1, 557-559.

(19) Nilsson, O.; Norberg, C. H.; Bergmark, J. E.; Fahlman, A.; Nordling, C.; Siegbahn, K. CHEMICAL SHIFTS IN EUROPIUM STUDIED BY ESCA. Helvetica Physica Acta 1968, 41, 1064-\&.

(20) Nordberg, R.; Albridge, R. G.; Bergmark, T.; Ericson, U.; Hedman, J.; Nordling, C.; Siegbahn, K.; Lindberg, B. J. MOLECULAR SPECTROSCOPY BY MEANS OF ESCA - CHARGE DISTRIBUTION IN NITROGEN COMPOUNDS. Arkiv for Kemi 1968, 28, 257-\&.

(21) Gelius, U.; Allan, C. J.; Johansso.G; Siegbahn, H.; Allison, D. A.; Siegbahn, K. ESCA SPECTRA OF BENZENE AND ISO-ELECTRONIC SERIES, THIOPHENE, PYRROLE AND FURAN. Physica Scripta $1971,3,237-\&$.

(22) Gelius, U.; Siegbahn, K. ESCA STUDIES OF MOLECULAR CORE AND VALENCE LEVELS IN GAS-PHASE. Faraday Discussions 1972, 54, 257-268.

(23) Gelius, U.; Asplund, L.; Basilier, E.; Hedman, S.; Helenelund, K.; Siegbahn, K. A HIGHRESOLUTION MULTIPURPOSE ESCA INSTRUMENT WITH X-RAY MONOCHROMATOR. Nuclear Instruments \& Methods in Physics Research Section B-Beam Interactions with Materials and Atoms 1984, 229, 85117.

(24) Saethre, L. J.; Martensson, N.; Svensson, S.; Malmquist, P. A.; Gelius, U.; Siegbahn, K. GAS-PHASE ESCA STUDIES OF 2,5-DIAZA-1,6-DIOXA-6A-THIAPENTALENE AND ITS SELENIUM AND TELLURIUM ANALOGS. Journal of the American Chemical Society 1980, 102, 1783-1788. 
(25) Ogletree, D. F.; Bluhm, H.; Lebedev, G.; Fadley, C. S.; Hussain, Z.; Salmeron, M. A differentially pumped electrostatic lens system for photoemission studies in the millibar range. Review of Scientific Instruments 2002, 73, 3872-3877.

(26) Salmeron, M.; Schlogl, R. Ambient pressure photoelectron spectroscopy: A new tool for surface science and nanotechnology. Surface Science Reports 2008, 63, 169-199.

-. This review articles discussed the surface analytic techniques developed in the last two decades. It exemplified main applications of these surface analytical techniques.

(27) Tao, F. Design of an in-house ambient pressure AP-XPS using a bench-top X-ray source and the surface chemistry of ceria under reaction conditions. Chemical Communications 2012, 48, 38123814.

(28) Tao, L. N. F. F. Development of a reaction cell for in-situ/operando studies of surface of a catalyst under a reaction condition and during catalysis Review of Scientific Instruments 2016, accepted.

(29) Grant, D. B. J. T.: Surface Analysis by Auger and X-Ray Photoelectron Spectroscopy; IMPublications, 2003.

(30) Zhang, S.; Shan, J.; Zhu, Y.; Nguyen, L.; Huang, W.; Yoshida, H.; Takeda, S.; Tao, F. Restructuring Transition Metal Oxide Nanorods for $100 \%$ Selectivity in Reduction of Nitric Oxide with Carbon Monoxide. Nano Letters 2013, 13, 3310-3314.

(31) Langell, M. A.; Kim, J. G.; Pugmire, D. L.; McCarroll, W. Nature of oxygen at rocksalt and spinel oxide surfaces. Journal of Vacuum Science \& Technology a-Vacuum Surfaces and Films 2001, 19, 1977-1982.

(32) Chuang, T. J.; Brundle, C. R.; Rice, D. W. INTERPRETATION OF X-RAY PHOTOEMISSION SPECTRA OF COBALT OXIDES AND COBALT OXIDE SURFACES. Surface Science 1976, 59, 413-429.

(33) Tao, F.; Grass, M. E.; Zhang, Y.; Butcher, D. R.; Renzas, J. R.; Liu, Z.; Chung, J. Y.; Mun, B. S.; Salmeron, M.; Somorjai, G. A. Reaction-Driven Restructuring of Rh-Pd and Pt-Pd Core-Shell Nanoparticles. Science 2008, 322, 932-934.

(34) Tao, F.; Grass, M. E.; Zhang, Y.; Butcher, D. R.; Aksoy, F.; Aloni, S.; Altoe, V.; Alayoglu, S.; Renzas, J. R.; Tsung, C.-K.; Zhu, Z.; Liu, Z.; Salmeron, M.; Somorjai, G. A. Evolution of Structure and Chemistry of Bimetallic Nanoparticle Catalysts under Reaction Conditions. Journal of the American Chemical Society 2010, 132, 8697-8703.

(35) Luan, N.; Zhang, S.; Yoon, S. J.; Tao, F. Preferential Oxidation of CO in H-2 on Pure Co3O4-X and Pt/Co3O4-x. Chemcatchem 2015, 7, 2346-2353.

(36) Ye, Y.; Wang, L.; Zhang, S.; Zhu, Y.; Shan, J.; Tao, F. The role of copper in catalytic performance of a Fe-Cu-Al-O catalyst for water gas shift reaction. Chemical Communications 2013, 49, 4385-4387.

(37) Shan, J.; Zhu, Y.; Zhang, S.; Zhu, T.; Rouvimov, S.; Tao, F. Catalytic Performance and in Situ Surface Chemistry of Pure alpha-MnO2 Nanorods in Selective Reduction of NO and N2O with CO. Journal of Physical Chemistry C 2013, 117, 8329-8335.

(38) Zhu, Y.; Zhang, S.; Ye, Y.; Zhang, X.; Wang, L.; Zhu, W.; Cheng, F.; Tao, F. Catalytic Conversion of Carbon Dioxide to Methane on Ruthenium-Cobalt Bimetallic Nanocatalysts and Correlation between Surface Chemistry of Catalysts under Reaction Conditions and Catalytic Performances. Acs Catalysis 2012, 2, 2403-2408.

(39) Wen, C.; Zhu, Y.; Ye, Y.; Zhang, S.; Cheng, F.; Liu, Y.; Wang, P.; Tao, F. Water-Gas Shift Reaction on Metal Nanoclusters Encapsulated in Mesoporous Ceria Studied with Ambient-Pressure X-ray Photoelectron Spectroscopy. Acs Nano 2012, 6, 9305-9313.

(40) Zeng, S.; Luan, N.; Cheng, F.; Liu, L.; Yu, Y.; Tao, F. Surface structure and chemistry of $\mathrm{Pt} / \mathrm{Cu} / \mathrm{Pt}(111)$ near surface alloy model catalyst in CO. Applied Surface Science 2014, 320, 225-230.

(41) Shan, J.-j.; Luan, N.; Zhang, S.; Tao, F.-F. Water-Gas Shift on Pd/alpha-MnO2 and Pt/alpha-MnO2. Catalysis Letters 2015, 145, 1571-1580. 